\title{
The Extended Operations for Generalized Quadratic Fuzzy Sets
}

\author{
Yong Sik Yun ${ }^{1}$ and Jin Won Park ${ }^{2}$ \\ 1 Department of Mathematics, Jeju National University, Korea \\ 2 Department of Mathematics Education, Jeju National University, Korea
}

\begin{abstract}
The extended algebraic operations are defined by applying the extension principle to normal algebraic operations. And these operations are calculated for some kinds of fuzzy numbers. In this paper, we get exact membership function as a results of calculation of these operations for generalized quadratic fuzzy sets.
\end{abstract}

Key Words : extension principle, generalized quadratic fuzzy set

\section{Introduction}

One of the most basic concepts of fuzzy set theory which can be used to generalize crisp mathematical concepts to fuzzy sets is the extension principle. Dubois and Prade defined the extended algebraic operations by applying the extension principle to normal algebraic operations, addition, subtraction, multiplication and division. And they get some results on the calculations of these operations for fuzzy triangular numbers.

In this paper, we define the generalized quadratic fuzzy set as a generalization of quadratic fuzzy set. And we get exact membership function as a results of calculation of these operations for generalized quadratic fuzzy sets.

\section{Preliminaries}

Following Zadeh, Dubois and Prade, the extension principle is defined as follows:

Definition 2.1. ([3]) Let $X=X_{1} \times \cdots \times X_{n}$ be a cartesian product and $\mu_{i}$ be a fuzzy set in $X_{i}$, respectively, and $f: X \rightarrow Y$ be a mapping. Then the extension principle allows us to define a fuzzy set $\nu$ in $Y$ by

$\nu(y)=\left\{\begin{array}{c}\sup _{\left(x_{1}, \cdots, x_{n}\right) \in f^{-1}(y)} \min \left\{\mu_{1}\left(x_{1}\right), \cdots, \mu_{n}\left(x_{n}\right)\right\} \\ \left(f^{-1}(y) \neq \emptyset\right) \\ 0 \quad\left(f^{-1}(y)=\emptyset\right) .\end{array}\right.$

For $n=1$, the extension principle reduces to a fuzzy set $\nu=f(\mu)$ defined by

접수일자 : 2010년 5월 17일

완료일자 : 2010년 6월 20일 $\nu(y)= \begin{cases}\sup _{x \in f^{-1}(y)} \mu(x) & \left(f^{-1}(y) \neq \emptyset\right) \\ 0 & \left(f^{-1}(y)=\emptyset\right) .\end{cases}$

Applying the extension principle to algebraic operations for fuzzy sets, we have the following definitions for extended operations.

Definition 2.2. ([3]) The extended addition, extended subtraction, extended multiplication, and extended division are defined by

(1) Addition $A(+) B$ :

$\mu_{A(+) B}(z)=\sup _{z=x+y} \min \left\{\mu_{A}(x), \mu_{B}(y)\right\}, x \in A, y \in B$.

(2) Subtraction $A(-) B$ :

$\mu_{A(-) B}(z)=\sup _{z=x-y} \min \left\{\mu_{A}(x), \mu_{B}(y)\right\}, x \in A, y \in B$.

(3) Multiplication $A(\cdot) B$ :

$\mu_{A(\cdot) B}(z)=\sup _{z=x \cdot y} \min \left\{\mu_{A}(x), \mu_{B}(y)\right\}, x \in A, y \in B$.

(4) Division $A(/) B$ :

$\mu_{A(/) B}(z)=\sup _{z=x / y} \min \left\{\mu_{A}(x), \mu_{B}(y)\right\}, x \in A, y \in B$.

For the calculations, we use the concepts of $\alpha$-cut.

Definition 2.3. The set $A_{\alpha}=\left\{x \in X \mid \mu_{A}(x) \geq \alpha\right\}$ is said to be the $\alpha$-cut of a fuzzy set $A$.

The membership function of a fuzzy set $A$ can be expressed in terms of the characteristic functions of its $\alpha$-cuts according to the formula

$$
\mu_{A}(x)=\sup _{\alpha \in(0,1]} \min \left(\alpha, \mu_{A_{\alpha}}(x)\right),
$$

where

$$
\mu_{A_{\alpha}}(x)= \begin{cases}1 & \left(x \in A_{\alpha}\right) \\ 0 & \text { (otherwise) }\end{cases}
$$


Remark ([1]) Let $A$ and $B$ be fuzzy sets and $A_{\alpha}=$ $\left[a_{1}^{(\alpha)}, a_{2}^{(\alpha)}\right]$ and $B_{\alpha}=\left[b_{1}^{(\alpha)}, b_{2}^{(\alpha)}\right]$ be the $\alpha$-cuts of $\mathrm{A}$ and $\mathrm{B}$, respectively. Then the $\alpha$-cuts of $A(+) B, A(-) B, A(\cdot) B$ and $A(/) B$ can be calculated as follows.
(1) $(A(+) B)_{\alpha}=A_{\alpha}(+) B_{\alpha}=\left[a_{1}^{(\alpha)}+b_{1}^{(\alpha)}, a_{2}^{(\alpha)}+b_{2}^{(\alpha)}\right]$.
(2) $(A(-) B)_{\alpha}=A_{\alpha}(-) B_{\alpha}=\left[a_{1}^{(\alpha)}-b_{2}^{(\alpha)}, a_{2}^{(\alpha)}-b_{1}^{(\alpha)}\right]$.
(3) $(A(\cdot) B)_{\alpha}=A_{\alpha}(\cdot) B_{\alpha}=\left[a_{1}^{(\alpha)} b_{1}^{(\alpha)}, a_{2}^{(\alpha)} b_{2}^{(\alpha)}\right]$.
(4) $(A(/) B)_{\alpha}=A_{\alpha}(/) B_{\alpha}=\left[a_{1}^{(\alpha)} / b_{2}^{(\alpha)}, a_{2}^{(\alpha)} / b_{1}^{(\alpha)}\right]$.

\section{Generalized quadratic fuzzy sets}

In this section, we define the generalized quadratic fuzzy sets as a generalization of quadratic fuzzy set and and apply the extended algebraic operations to these fuzzy sets.

Definition 3.1. A fuzzy set $A$ with a membership function

$$
\mu_{A}(x)= \begin{cases}0 & \left(x<x_{1}, x_{2} \leq x\right) \\ -a\left(x-x_{1}\right)\left(x-x_{2}\right) & \\ =-a(x-m)^{2}+p & \left(x_{1} \leq x<x_{2}\right)\end{cases}
$$

where $0<a$ and $0<p \leq 1$ is called a generalized quadratic fuzzy set and denoted by $\left[\left[x_{1}, p, x_{2}\right]\right]$ or $[[a, m, p]]_{+}$.

Theorem 3.2. Let $A=\left[\left[x_{1}, p, x_{2}\right]\right]=[[a, m, p]]_{+}$and $B=\left[\left[x_{3}, q, x_{4}\right]\right]=[[b, n, q]]_{+}$be generalized quadratic fuzzy sets. Suppose $p \leq q$ and $\mu_{B}(x) \geq p$ on $\left[k_{1}, k_{2}\right]$. Then we have the followings.

(1) $A(+) B$ is a fuzzy set with a membership function

$$
\mu_{A(+) B}(x)= \begin{cases}0 & \left(x<x_{1}+x_{3}, x_{2}+x_{4} \leq x\right) \\ f_{1}(x) & \left(x_{1}+x_{3} \leq x<m+k_{1}\right) \\ p & \left(m+k_{1} \leq x<m+k_{2}\right) \\ f_{2}(x) & \left(m+k_{2} \leq x<x_{2}+x_{4}\right)\end{cases}
$$

where

$$
\begin{aligned}
f_{1}(x) & =\frac{1}{a^{2}-2 a b+b^{2}}(-a b m(a+b+a n+b n) \\
& -a b n(a m+b m+a n+b n)-a b(p+q) \\
& +a^{2} q+b^{2} p+2 a b(a m+b m+a n+b n) x \\
& \left.-a b(a+b) x^{2}+2 a b(m+n-x) \cdot \sqrt{g(x)}\right)
\end{aligned}
$$

and

$$
\begin{aligned}
f_{2}(x) & =\frac{1}{a^{2}-2 a b+b^{2}}(-a b m(a+b+a n+b n) \\
& -a b n(a m+b m+a n+b n)-a b(p+q) \\
& +a^{2} q+b^{2} p+2 a b(a m+b m+a n+b n) x \\
& \left.-a b(a+b) x^{2}-2 a b(m+n-x) \cdot \sqrt{g(x)}\right),
\end{aligned}
$$

where $g(x)=a b(m+n)^{2}+(a-b)(p-q)-2 a b(m+$ n) $x+a b x^{2}$.

(2) $A(-) B$ is a fuzzy set with a membership function

$$
\mu_{A(-) B}(x)= \begin{cases}0 & \left(x<x_{1}-x_{4}, x_{2}-x_{3} \leq x\right) \\ f_{1}(x) & \left(x_{1}-x_{4} \leq x<m-k_{2}\right) \\ p & \left(m-k_{2} \leq x<m-k_{1}\right) \\ f_{2}(x) & \left(m-k_{1} \leq x<x_{2}-x_{3}\right)\end{cases}
$$

where

$$
\begin{aligned}
f_{1}(x) & =\frac{1}{a^{2}-2 a b+b^{2}}(-a b m(a m+b m-a n-b n) \\
& -a b n(a n+b n-a m-b m)-a b(p+q) \\
& +a^{2} q+b^{2} p+2 a b(a m+b m-a n-b n) x \\
& \left.-a b^{2} x^{2}+2 a b(m-n-x) \cdot \sqrt{g(x)}\right)
\end{aligned}
$$

and

$$
\begin{aligned}
f_{2}(x) & =\frac{1}{a^{2}-2 a b+b^{2}}(-a b m(a m+b m-a n-b n) \\
& -a b n(a n+b n-a m-b m)-a b(p+q) \\
& +a^{2} q+b^{2} p+2 a b(a m+b m-a n-b n) x \\
& \left.-a b^{2} x^{2}-2 a b(m-n-x) \cdot \sqrt{g(x)}\right),
\end{aligned}
$$

where $g(x)=a b(m-n)^{2}+(a-b)(p-q)-2 a b(m-$ $n) x+a b x^{2}$.

(3) If $p=q, A(\cdot) B$ is a fuzzy set with a membership function

$$
\mu_{A(\cdot) B}(x)= \begin{cases}0 & \left(x<x_{1} x_{3}, x_{2} x_{4} \leq x\right) \\ f(x) & \left(x_{1} x_{3} \leq x<x_{2} x_{4}\right)\end{cases}
$$

where

$$
f(x)=\frac{1}{2}\left(-a m^{2}-b n^{2}+2 p\right)-\sqrt{a b} x+\frac{1}{2} \sqrt{g(x)},
$$

and

$$
\begin{aligned}
g(x) & =-a m^{2}\left(a m^{2}+3 b n^{2}\right)-b n^{2}\left(b n^{2}+3 a m^{2}\right) \\
& +2\left(a m^{2}+b n^{2}-2 p\right)^{2}+8 p\left(a m^{2}+b n^{2}-p\right) \\
& +8 a b m n x-\frac{1}{8 \sqrt{a b} x}\left\{-8\left(a m^{2}+b n^{2}-2 p\right)^{3}\right. \\
& \left.+8\left(a m^{2}+b n^{2}-2 p\right) h_{1}(x)-16 h_{2}(x)\right\},
\end{aligned}
$$

and where

$$
\begin{aligned}
h_{1}(x) & =a m^{2}\left(a m^{2}+2 b n^{2}\right)+b n^{2}\left(b n^{2}+2 a m^{2}\right) \\
& -6 p\left(a m^{2}+b n^{2}-p\right)-4 a b m n x-2 a b x^{2}
\end{aligned}
$$

and

$$
\begin{aligned}
& h_{2}(x)=a b m^{2} n^{2}\left(a m^{2}+b n^{2}-4 p\right)-a m^{2} p\left(a m^{2}\right. \\
& -3 p)-b n^{2} p\left(b n^{2}-3 p\right)-2 p^{3}-2 a b m n \\
& \left(a m^{2}+b n^{2}-2 p\right) x+a b\left(a m^{2}+b n^{2}+2 p\right) x^{2} .
\end{aligned}
$$


(4) $A(/) B$ is a fuzzy set with a membership function

$$
\mu_{A(/) B}(x)= \begin{cases}0 & \left(x<x_{1} / x_{4}, x_{2} / x_{3} \leq x\right) \\ f_{1}(x) & \left(x_{1} / x_{4} \leq x<m / k_{2}\right) \\ p & \left(m / k_{2} \leq x<m / k_{1}\right) \\ f_{2}(x) & \left(m / k_{1} \leq x<x_{2} / x_{3}\right)\end{cases}
$$

where

$$
\begin{aligned}
f_{1}(x) & =\frac{1}{b^{2}-2 a b x^{2}+a^{2} x^{4}}\left(-b^{2}\left(a m^{2}+p\right)\right. \\
& +2 a b^{2} m n x-a b\left(a m^{2}+b n^{2}+p+q\right) x^{2} \\
& +2 a^{2} b m n x^{3}-a^{2}\left(b n^{2}-q\right) x^{4} \\
& +2 a b x(m-n x) \cdot \sqrt{g(x)})
\end{aligned}
$$

and

$$
\begin{aligned}
f_{2}(x) & =\frac{1}{b^{2}-2 a b x^{2}+a^{2} x^{4}}\left(-b^{2}\left(a m^{2}+p\right)\right. \\
& +2 a b^{2} m n x-a b\left(a m^{2}+b n^{2}+p+q\right) x^{2} \\
& +2 a^{2} b m n x^{3}-a^{2}\left(b n^{2}-q\right) x^{4} \\
& -2 a b x(m-n x) \cdot \sqrt{g(x)})
\end{aligned}
$$

and where $g(x)=b\left(a m^{2}-p+q\right)-2 a b m n x+a\left(b n^{2}+\right.$ $p-q) x^{2}$.

Proof. It is enough to calculate the $\alpha$-cut of $A(+) B$, $A(-) B, A(\cdot) B$ and $A(/) B$. Note that

$$
\mu_{A}(x)= \begin{cases}0 & \left(x<x_{1}, \quad x_{2} \leq x\right) \\ -a\left(x-x_{1}\right)\left(x-x_{2}\right) & \\ =-a(x-m)^{2}+p & \left(x_{1} \leq x<x_{2}\right)\end{cases}
$$

and

$$
\mu_{B}(x)= \begin{cases}0 & \left(x<x_{3}, \quad x_{4} \leq x\right) \\ -b\left(x-x_{3}\right)\left(x-x_{4}\right) & \\ =-b(x-n)^{2}+q & \left(x_{3} \leq x<x_{4}\right)\end{cases}
$$

Let $A_{\alpha}=\left[a_{1}^{(\alpha)}, a_{2}^{(\alpha)}\right]$ and $B_{\alpha}=\left[b_{1}^{(\alpha)}, b_{2}^{(\alpha)}\right]$ be the $\alpha$ cuts of $A$ and $B$, respectively. Since $\alpha=-a\left(a_{1}^{(\alpha)}-m\right)^{2}+$ $p=-a\left(a_{2}^{(\alpha)}-m\right)^{2}+p$, we have

$$
A_{\alpha}=\left[a_{1}^{(\alpha)}, a_{2}^{(\alpha)}\right]=\left[m-\sqrt{\frac{p-\alpha}{a}}, m+\sqrt{\frac{p-\alpha}{a}}\right] .
$$

Similarly, we have

$$
B_{\alpha}=\left[b_{1}^{(\alpha)}, b_{2}^{(\alpha)}\right]=\left[n-\sqrt{\frac{q-\alpha}{b}}, n+\sqrt{\frac{q-\alpha}{b}}\right] .
$$

(1) Since $p \leq q$ and $\mu_{B}(x) \geq p$ on $\left[k_{1}, k_{2}\right], \mu_{A(+) B}(x)=$ $p$ if $x \in\left[m+k_{1}, m+k_{2}\right]$. By the above facts,

$$
\begin{aligned}
A_{\alpha}(+) B_{\alpha}= & {\left[a_{1}^{(\alpha)}+b_{1}^{(\alpha)}, a_{2}^{(\alpha)}+b_{2}^{(\alpha)}\right] } \\
= & {\left[m-\sqrt{\frac{p-\alpha}{a}}+n-\sqrt{\frac{q-\alpha}{b}},\right.} \\
& \left.m+\sqrt{\frac{p-\alpha}{a}}+n+\sqrt{\frac{q-\alpha}{b}}\right] .
\end{aligned}
$$

If $x \in\left[x_{1}+x_{3}, m+k_{1}\right]$, then $m-\sqrt{\frac{p-\alpha}{a}}+n-\sqrt{\frac{q-\alpha}{b}}=x$. Hence $\alpha=f_{1}(x)$. Similarly, for $x \in\left[m+k_{2}, x_{2}+x_{4}\right]$, we have $\alpha=f_{2}(x)$.

(2) Since $p \leq q$ and $\mu_{B}(x) \geq p$ on $\left[k_{1}, k_{2}\right], \mu_{A(-) B}(x)=$ $p$ if $x \in\left[m-k_{2}, m-k_{1}\right]$. By the above facts,

$$
\begin{aligned}
A_{\alpha}(-) B_{\alpha}= & {\left[a_{1}^{(\alpha)}-b_{2}^{(\alpha)}, a_{2}^{(\alpha)}-b_{1}^{(\alpha)}\right] } \\
= & {\left[m-\sqrt{\frac{p-\alpha}{a}}-\left(n+\sqrt{\frac{q-\alpha}{b}}\right)\right.} \\
& \left.m+\sqrt{\frac{p-\alpha}{a}}-\left(n-\sqrt{\frac{q-\alpha}{b}}\right)\right] .
\end{aligned}
$$

If $x \in\left[x_{1}-x_{4}, m-k_{2}\right]$, then $m-\sqrt{\frac{p-\alpha}{a}}-\left(n+\sqrt{\frac{q-\alpha}{b}}\right)=$ $x$. Hence $\alpha=f_{1}(x)$. Similarly, for $x \in\left[m-k_{1}, x_{2}-x_{3}\right]$, we have $\alpha=f_{2}(x)$.

(3) Suppose $p=q$. Then $\mu_{A}(m)=\mu_{B}(n)=p$ and so $\mu_{A(\cdot) B}(m n)=p$. By the above facts,

$$
\begin{aligned}
A_{\alpha}(\cdot) B_{\alpha}= & {\left[a_{1}^{(\alpha)} b_{1}^{(\alpha)}, a_{2}^{(\alpha)} b_{2}^{(\alpha)}\right] } \\
= & {\left[\left(m-\sqrt{\frac{p-\alpha}{a}}\right)\left(n-\sqrt{\frac{p-\alpha}{b}}\right),\right.} \\
& \left.\left(m+\sqrt{\frac{p-\alpha}{a}}\right)\left(n+\sqrt{\frac{p-\alpha}{b}}\right)\right] .
\end{aligned}
$$

Since $\left(m-\sqrt{\frac{p-\alpha}{a}}\right)\left(n-\sqrt{\frac{p-\alpha}{b}}\right)=x$ or $(m+$ $\left.\sqrt{\frac{p-\alpha}{a}}\right)\left(n+\sqrt{\frac{p-\alpha}{b}}\right)=x$, we have $\alpha=f(x)$.

(4) Since $p \leq q$ and $\mu_{B}(x) \geq p$ on $\left[k_{1}, k_{2}\right], \mu_{A(/) B}(x)=p$ if $x \in\left[m / k_{2}, m / k_{1}\right]$. By the above facts,

$$
\begin{aligned}
A_{\alpha}(/) B_{\alpha}= & {\left[a_{1}^{(\alpha)} / b_{2}^{(\alpha)}, a_{2}^{(\alpha)} / b_{1}^{(\alpha)}\right] } \\
= & {\left[\left(m-\sqrt{\frac{p-\alpha}{a}}\right) /\left(n+\sqrt{\frac{q-\alpha}{b}}\right)\right.} \\
& \left.\left(m+\sqrt{\frac{p-\alpha}{a}}\right) /\left(n-\sqrt{\frac{q-\alpha}{b}}\right)\right] .
\end{aligned}
$$

If $x \in\left[\frac{x_{1}}{x_{4}}, \frac{m}{k_{2}}\right]$, then $\left(m-\sqrt{\frac{p-\alpha}{a}}\right) /\left(n+\sqrt{\frac{q-\alpha}{b}}\right)=x$. Hence $\alpha=f_{1}(x)$. Similarly, for $x \in\left[\frac{m}{k_{1}}, \frac{x_{2}}{x_{3}}\right]$, we have $\alpha=f_{2}(x)$.

Remark In case of extended multiplication, if $p \neq q$, the membership function of $A(\cdot) B$ contains so many terms and so we did not write down the explicit form. 
Example 3.3. Let $A=\left[\left[2, \frac{2}{3}, 8\right]\right]$ and $B=\left[\left[3, \frac{3}{4}, 11\right]\right]$. Then we have the followings.

(1) The extended addition reduces to

$$
\mu_{A(+) B}(x)= \begin{cases}0 & (x<5,19 \leq x) \\ f_{1}(x) & \left(5 \leq x<\frac{32}{3}\right) \\ 2 / 3 & \left(\frac{32}{3} \leq x<\frac{40}{3}\right) \\ f_{2}(x) & \left(\frac{40}{3} \leq x<19\right)\end{cases}
$$

where

$$
\begin{aligned}
f_{1}(x)= & \frac{1}{4418}\left(-357204+60192 x-2508 x^{2}\right. \\
& \left.+288(x-12) \sqrt{10321-1728 x+72 x^{2}}\right)
\end{aligned}
$$

and

$$
\begin{aligned}
f_{2}(x)= & \frac{1}{4418}\left(-357204+60192 x-2508 x^{2}\right. \\
& \left.-288(x-12) \sqrt{10321-1728 x+72 x^{2}}\right) .
\end{aligned}
$$

(2) The extended subtraction reduces to

$$
\mu_{A(-) B}(x)= \begin{cases}0 & (x<-9,5 \leq x) \\ f_{1}(x) & \left(-9 \leq x<\frac{-10}{3}\right) \\ 2 / 3 & \left(\frac{-10}{3} \leq x<\frac{-2}{3}\right) \\ f_{2}(x) & \left(\frac{-2}{3} \leq x<5\right)\end{cases}
$$

where

$$
\begin{aligned}
f_{1}(x)= & \frac{-1}{4418}\left(6084+10032 x+2508 x^{2}\right. \\
& \left.+288(x+2) \sqrt{241+288 x+72 x^{2}}\right)
\end{aligned}
$$

and

$$
\begin{aligned}
f_{2}(x)= & \frac{-1}{4418}\left(6084+10032 x+2508 x^{2}\right. \\
& \left.-288(x+2) \sqrt{241+288 x+72 x^{2}}\right) .
\end{aligned}
$$

Example 3.4. Let $A=\left[\left[2, \frac{2}{3}, 8\right]\right]$ and $B=\left[\left[3, \frac{2}{3}, 11\right]\right]$. Then we have the followings.

(1) The extended multiplication reduces to

$$
\mu_{A(\cdot) B}(x)= \begin{cases}0 & (x<6,88 \leq x) \\ f(x) & (6 \leq x<88)\end{cases}
$$

where $f(x)=\frac{1}{432}(-553-24 x+41 \sqrt{1+48 x})$.
(2) The extended division reduces to

$$
\mu_{A(/) B}(x)= \begin{cases}0 & \left(x<\frac{2}{11}, \frac{8}{3} \leq x\right) \\ f_{1}(x) & \left(\frac{2}{11} \leq x<\frac{3}{5}\right) \\ 2 / 3 & \left(\frac{3}{5} \leq x<\frac{15}{17}\right) \\ f_{2}(x) & \left(\frac{15}{17} \leq x<\frac{8}{3}\right)\end{cases}
$$

where

$$
\begin{aligned}
& f_{1}(x)=\frac{-1}{6561-20736 x^{2}+16384 x^{4}}(7776-34020 x \\
& +57702 x^{2}-53760 x^{3}+25344 x^{4} \\
& \left.+144 x(7 x-5) \sqrt{1881-5040 x+3400 x^{2}}\right)
\end{aligned}
$$

and

$$
\begin{aligned}
& f_{2}(x)=\frac{-1}{6561-20736 x^{2}+16384 x^{4}}(7776-34020 x \\
& +57702 x^{2}-53760 x^{3}+25344 x^{4} \\
& \left.-144 x(7 x-5) \sqrt{1881-5040 x+3400 x^{2}}\right) \text {. }
\end{aligned}
$$

\section{References}

[1] A. Kaufmann and M.M. Gupta, Introduction To Fuzzy Arithmetic: Theory and Applications, Van Nostrand Reinhold Co., New York, 1985.

[2] Y.S. Yun, S.U. Ryu and J.W. Park, ”The generalized triangular fuzzy sets," Journal of the Chungcheong Mathematical Society, vol. 22, no. 2, pp. 161-170, 2009.

[3] H.J. Zimmermann, Fuzzy set Theory - and Its Applications, Kluwer-Nijhoff Publishing, BostonDordrecht-Lancaster, 1985.

\section{저 자 소 개}

\section{Yong Sik Yun}

Professor of Jeju National University

Research Area: Fuzzy probability, Probability theory E-mail : yunys@jejunu.ac.kr

\section{Jin Won Park}

Professor of Jeju National University Research Area: Fuzzy probability, General topology

E-mail : jinwon@jejunu.ac.kr 